\title{
A MONITORIA ACADÊMICA DA DISCIPLINA DE CUIDADOS CRÍTICOS PARA A ENFERMAGEM: UM RELATO DE EXPERIÊNCIA
}

\author{
Lívia Dourado MAGALHÃES ${ }^{1}$ \\ Ionara de Souza JANUÁRIO
}

Anna Karla Fausto MAIA ${ }^{3}$

\begin{abstract}
${ }^{1}$ Discente do Curso Bacharelado em Enfermagem da Universidade Federal de Campina Grande e Bolsitado Programa de Monitoria da Disciplina de Cuidados Críticos - Vigência - 2013 - UFCG. E-mail: liviamagalhaes jd@ @hotmail.com

${ }^{2}$ Discente do Curso Bacharelado em Enfermagem da Universidade Federal de Campina Grande e Voluntária do Programa de Monitoria da Disciplina de Cuidados Críticos - Vigência - 2013 - UFCG. E-mail: ionara76@hotmail.com
\end{abstract}

${ }^{3}$ Enf. Esp. Em assistência e Gestão em Saúde da Família e Auditoria. Mestranda em Gestão Hospitalar e Saúde Coletiva pela Faculdade do Norte do Paraná. E-mail: akarla-maia@ hotmail.com

Recebido em: 30/05/2014 - Aprovado em: 25/09/2014 - Disponibilizado em: 15/12/2014

\begin{abstract}
RESUMO
OBJETIVO: Relatar a experiência dos monitores da disciplina de Cuidados Críticos do curso Bacharelado em Enfermagem no desempenho de suas atividades acadêmicas, no período compreendido entre os meses de maio de 2013 a abril de 2014. METODOLOGIA: Trata-se de um relato de experiência apresentando as vivências dos monitores da disciplina de Cuidados Críticos da Universidade Federal de Campina Grande, campus de Cuité, no período referente ao semestre 2013.1 e 2013.2. RESULTADOS E DISCUSSÃO: Durante a vigência da monitoria foram realizadas atividades no laboratório da disciplina, bem como na sala de monitoria, que tinha por finalidade esclarecer possíveis dúvidas dos discentes sobre os assuntos abordados em sala de aula pelo professor. Ao decorrer dos períodos em que ocorreu a monitoria 68 alunos foram beneficiados, 30 alunos no período 2013.1 com aprovação de 27 alunos e 38 alunos no período 2013.2, destes todos foram aprovados. A relevância da monitoria acadêmica no ensino superior vai além da aquisição de um título curricular, além de fomentar um ganho intelectual no aspecto pessoal do monitor, ela contribui substancialmente para o conhecimento dos alunos monitorados e, especialmente, na relação entre professor orientador e aluno monitor, onde favorece a troca de conhecimentos. CONCLUSÃO: A monitoria ao longo dos dois períodos contribuiu para versar a capacidade dos monitores para a concentração, argumentação e domínio sobre o grupo. Ressalta-se também a importância da elaboração dos estudos e pesquisas realizadas, e das trocas de conhecimentos que contribuiu para o engrandecimento intelectual e social dos monitores, revelando assim novas perspectivas profissional.
\end{abstract}

Descritores: Enfermagem. Ensino. Aprendizagem. Estudantes de Enfermagem. Cuidados Críticos.

\begin{abstract}
OBJECTIVE: To report our experience monitors the discipline of Critical Care Bachelor of Nursing course in performing their academic activities in the period between the months of May 2013 to April 2014. METHODOLOGY: This is an experience report presenting the experiences of the monitors of the discipline of Critical Care, Federal University of Campina Grande, campus Cuité, the period for the six 2013.1 and 2013.2. RESULTS AND DISCUSSION: During the term of the monitoring activities were conducted in the laboratory discipline as well as in the monitoring room, which was intended to clarify possible doubts of students on the topics discussed in the classroom by the teacher. During the periods in which the monitoring occurred 68 students were benefited 30 students in the period 2013.1 with approval of 27 students and 38 students in the period 2013.2, all of these were approved. The relevance of academic monitoring in higher education goes beyond the acquisition of a curriculum title, as well as fostering an intellectual gain the personal aspect of the monitor, it contributes substantially to the knowledge of the
\end{abstract}


students and monitored, especially in the relationship between academic advisor and monitor student, which promotes the exchange of knowledge. CONCLUSION: The monitoring over the two periods versar contributed to the ability of monitors to the concentration, reasoning and rule over the group. Also emphasized the importance of preparing the studies and research carried out, and the exchange of knowledge that contributed to the intellectual and social aggrandizement monitors, thus revealing new professional perspectives.

Keywords: Nursing. Education. Learning. Nursing students. Critical Care.

\section{INTRODUÇÃO}

Conforme Resolução N 311 de 2007, do Conselho Federal de Enfermagem que diz respeito ao Código de Ética dos Profissionais de Enfermagem, a Enfermagem é uma ciência que envolve uma gama de conhecimentos científicos e técnicos, desenvolvido a partir de práticas sociais, éticas e políticas que ocorrem por meio do ensino, pesquisa e assistência, atuando na prestação de serviços à pessoa, família e comunidade, de acordo com o contexto em que estão inseridos.

O curso de Bacharelado em Enfermagem da Universidade Federal de Campina Grande, do Centro de Educação e Saúde, está localizado no município de Cuité, estado da ParaíbaPB. O mesmo foi criado pela resolução No 09 de 2005 da Câmara Superior de Ensino do Conselho Universitário da Universidade Federal de Campina Grande (CSE/UFCG) em 22 de novembro de 2005, sendo esta reconhecida pelo Ministério da
Educação (RESOLUÇÃO CSE/UFCG N¹4 DE JUNHO DE 2007). A primeira estrutura curricular implantada possui uma carga horária mínima de 4.050 horas de atividades didáticas, correspondentes a 270 créditos, das quais, 90 horas destinam-se à disciplina de Cuidados Critícos (RESOLUÇÃO CSE/UFCG $\mathrm{N}^{\circ} 07$ DE ABRIL DE 2009).

A disciplina de Cuidados Críticos é de grande relevância no processo de formação do Enfermeiro, haja vista que ela deve atender ao princípio da integralidade da assistência, em todos os níveis de complexidade do sistema de saúde, mediante os desígnios dos modelos clínicos e epidemiológicos vigentes (LINO; CALIL, 2007).

\section{Conforme a Resolução COFEN} $\mathrm{N}^{\mathrm{o}} 293$ de setembro de 2004, a assistência prestada em Unidade de Cuidados Críticos ou Intensivos deve ser integral e especializada pela equipe de enfermagem e médica, voltada para aqueles pacientes graves, porém 
recuperáveis, com risco iminente de morte, sujeitos à instabilidade das funções vitais.

Observa-se que o cuidado prestado pela equipe de enfermagem na Unidade de Terapia Intensiva requer conhecimentos e habilidades específicas e que estes estejam capacitados para prestar cuidados em meio a um ambiente de aparelhagens múltiplas, desconforto, impessoalidade e dependência tecnológica (NASCIMENTO; TRENTINI, 2004). Nesse sentido, ressalta-se a importância de preparar os futuros profissionais de enfermagem durante a graduação, para que estes possam oferecer futuramente, um atendimento adequado e acertivo a pessoas em situações críticas de saúde e livre de erros e ocorrências de iatrogênias (LINO; CALIL, 2007).

O programa institucional de monitoria acadêmica é assegurado pela Lei de Diretrizes e Bases da Educação Nacional No 9.394, de 20 de Dezembro de 1996, que estabelece as diretrizes e bases da educação nacional, a qual permite aos acadêmicos da educação superior executar tarefas de ensino e pesquisa pelas respectivas instituições em que são vinculados, desempenhando funções de monitoria, de acordo com seu rendimento e plano de estudos (BRASIL, 1996).

O currículo da graduação no ensino superior é composto de ensino, pesquisa e extensão, sendo a monitoria incluída na categoria de pesquisa (SOUZA, 2009). A monitoria consiste em um serviço de apoio pedagógico que permite a consolidação da formação acadêmica, possibilitando aos discentes aprimorar seus conhecimentos e resolver possíveis dificuldades relacionadas à matéria trabalhada em sala de aula viabilizando desta forma uma melhor correlação entre teoria e prática, e consequentemente favorecendo ao processo de ensino e aprendizagem (CARVALHO et al., 2012).

A fim de contribuir para a reflexão e o fortalecimento das atividades de monitoria no cenário acadêmico, ao mesmo que provocar o estudo e discussão acerca da relevância deste tema entre a comunidade acadêmica percebeu-se a importância da realização deste estudo. Deste modo, o mesmo tem como objetivo relatar a experiência dos monitores da disciplina de Cuidados Críticos do curso Bacharelado em Enfermagem no desempenho de suas atividades 
acadêmicas, no período compreendido entre os meses de maio de 2013 a abril de 2014.

\section{METODOLOGIA}

Trata-se de um relato de experiência, acerca das vivências dos monitores da disciplina de Cuidados Críticos do Curso de Bacharelado em Enfermagem da Universidade Federal de Campina Grande (UFCG), campus de Cuité, no período referente aos semestres 2013.1 e 2013.2, que corresponde aos meses de maio de 2013 a abril de 2014.

Neste sentido, foi utilizado o método descritivo. De acordo com Cervo; Berviam; Da Silva (2007) a descrição observa, registra e analisa as características de uma população ou fenômeno, sem manipulá-las, através de técnicas padronizadas de coleta de dados, tais como a observação sistemática. Para tanto, realizou-se uma análise crítica das atividades desenvolvidas durante $o$ período subscrito como monitores da disciplina e dos relatórios desenvolvidos ao final de cada semestre letivo.

No âmbito da disciplina de Cuidados Críticos para Enfermagem, as atividades de monitoria foram desenvolvidas por alunos que já haviam cursardo a referida disciplina. Ademais, os estudantes foram aprovados em processo seletivo instituído no primeiro semestre de 2013, por intermédio da solicitação do Docente titular da disciplina, da diretoria acadêmica da UFCG em concordância com a coordenação do curso de enfermagem. O mesmo constituiu-se de prova escrita, entrevista com a Docente titular da disciplina e análise do rendimento acadêmico dos candidatos. Nesse processo, foram selecionados três monitores, sendo um bolsista e dois volntários, os quais posteriormente foram distribuídos entre grupos de cinco alunos, permanecendo cada monitor com dois grupos.

O relato de experiência está além de uma mera descrição sumária sobre alguma atividade, pois ao efetuar a sua leitura, é presumível conhecer com mais propriedade a experiência descrita. Possibilita ainda, do ponto de vista teórico, compará-la com outras experiências semelhantes, permitindo uma maior reflexão sobre a temática abordada. Desta forma, a realização deste estudo é justificável, uma vez que possibilitará uma maior discussão e 
ampliação de novos estudos sobre o tema trabalhado, além de fornecer subsídios para o desenvolvimento de futuras pesquisas que tenham a temática como foco centralizador.

\section{RESULTADOS E DISCUSSÃO}

A disciplina de Cuidados Críticos é ofertada para os acadêmicos de enfermagem matriculados no oitavo período, consta de uma de disciplina de seis créditos e carga horária de 90 horas. Destas, 75 horas são referentes a teoria e 15 horas dedicadas à prática. Além disto, a disciplina mencionada anteriormente tem como objetivo geral prestar assistência de enfermagem integral e sistematizada aos pacientes adultos e/ou idosos, criticamente enfermos e a suas famílias internados em Unidades de Terapia Intensiva (UTI), observando os aspectos éticohumanísticos, além de compreender e discutir o papel do enfermeiro nessas unidades. Esta disciplina ainda contempla como objetivos específicos apresentar assistência de enfermagem nos diversos sistemas, como cardíaco, respiratório, gastrointestinal, urinário, neurológico em pacientes críticos de terapia intensiva.
Conforme Edital $n^{\circ} \quad 17 / 2013$ estabelecido pela Pró Reitoria de Ensino (PRE) da UFCG, Item 4 são atribuições do monitor: executar atividades pedagógicas sob a orientação do professor; constituir elo entre professores e alunos, a fim de desenvolver a aprendizagem; participar, a critério do professor-orientador, das aulas ministradas da disciplina em que é monitor; colaborar com o professor na realização de trabalhos teóricos, práticos e experimentais, na preparação de material didático e em atividades de classe e/ou laboratório; contribuir com o professor na orientação de alunos, esclarecendo e tirando dúvidas nas atividades da disciplina; participar de atividades que propiciem o seu aprofundamento na disciplina, como a elaboração de trabalhos científicos e sua apresentação em encontros acadêmicos; elaborar o relatório de suas atividades ao final de cada período letivo.

As atividades realizadas pelos monitores da referida disciplina vão desde acompanhamento das aulas e atividades, orientação, esclarecimentos de dúvidas de alunos, e por tratar-se de uma disciplina que contempla carga horária prática com estágios a nível hospitalar, viu-se também a necessidade 
de realização de aulas práticas em laboratório com os monitores para que assim os alunos tivessem a oportunidade de realizar e revisar os procedimentos relacionados a disciplina. Para tanto, os dias e horários foram combinados previamente com os alunos para que assim tornassem mais acessíveis para os mesmos.

A instituição de ensino disponibilizou uma sala de monitoria para os encontros semanais, que tinha por finalidade esclarecer possíveis dúvidas dos discentes sobre os assuntos abordados em sala de aula pelo professor. Também foi disponibilizado o laboratório da disciplina equipado com materiais, aparelhos e manequins, onde os monitores acompanhavam as aulas práticas ministradas pelos docentes, assim como também dispunham de horários agendados distintos aos das aulas, para praticar e/ou elucidar as dúvidas acerca das aulas teóricas e práticas. Cabia também ao monitor preparar e organizar o laboratório disponibilizado para as práticas da disciplina ministradas pelo professor, bem como, organização dos materiais e instrumentais ofertados, e responsabilização pelos mesmos nos seus horários de monitoria.
Neste contexto, pôde-se perceber que os horários disponibilizados para as práticas em laboratório favorecem $\mathrm{o}$ aprendizado do aluno, pois os mesmos têm a oportunidade de simular a realização de procedimentos que rotineiramente fazem parte de sua vida profissional e, previamente desenvolvem suas habilidades manuais antes do contato direto com os pacientes no estágio realizado na rede hospitalar (CARVALHO et al., 2012).

Os monitores cumpriram uma carga horária obrigatória de 12 horas semanais de acordo com o Edital da PRE $N^{\circ}$ 17/2013, sendo 8 destas destinadas ao atendimento ao aluno na sala da monitoria ou no laboratório de Enfermagem e 4 horas destinadas ao estudo da disciplina. Os horários foram disponibilizados pelos 3 monitores ao longo da semana, nos turnos matutino, vespertino ou noturno. Foi notório entre os monitores a importância da realização contínua de estudos da disciplina, para que assim houvesse um bom aproveitamento do conteúdo e que o mesmo pudesse repassá-lo de forma mais proveitosa para os discentes que estavam cursando a disciplina.

A relevância da monitoria acadêmica no ensino superior vai além 
da aquisição de um título curricular, além de fomentar um ganho intelectual no aspecto pessoal do Monitor, ela contribui substancialmente para o conhecimento dos alunos monitorados e, especialmente, na relação entre professor orientador e aluno monitor, onde favorece a troca de conhecimentos (SOUZA, 2009).

Nesse interim, percebe-se que a monitoria acadêmica traz benefícios para monitores, professores e alunos, uma vez que possibilita a troca de conhecimentos entre os envolvidos, proporciona um maior contato entre $\mathrm{o}$ monitor e professor e serve como elo entre os discentes e monitores. Para os monitores, a monitoria possibilita um contato próximo com a docência, capacidade de desenvolver suas habilidades didático-pedagógicas, possibilidade de estudar e se aprofundar nos conteúdos para assim repassá-los. Os discentes também são favorecidos neste processo, uma vez que se sentem mais a vontade para sanar suas dúvidas e fazer questionamentos ao monitor.

Conforme Carvalho et al (2012) as aulas práticas que fazem parte da grade curricular obrigatória dos cursos de Enfermagem tem como objetivo subsidiar a formação do profissional, através das práticas que são realizadas diariamente em laboratórios ou unidades de saúde incentivando o aluno a enfrentar os problemas, tomar decisões e formar profissionais autônomos com capacidade de atuar nos mais diversos níveis de atenção a saúde, como por exemplo na UTI.

Corroborando com os resultados obtidos neste estudo, Cardoso; Araújo (2008) trazem ainda em suas pesquisas algumas contribuições da monitoria para a formação acadêmica e enquanto futuro profissional de Enfermagem, dentre elas: atuação dos monitores enquanto facilitadores do processo de ensino-aprendizagem; estímulo à docência; participação na construção de conhecimento e prática da monitoria como um adicional curricular, uma vez que o aluno monitor pode atuar diretamente no auxilio da formação em enfermagem, sendo, portanto um agente definidor de mudanças.

A monitoria proporciona ainda aperfeiçoamento das habilidades técnicas de enfermagem, incentiva a ética, a segurança, a capacidade de observação, conhecimento, estimula os alunos ao manuseio de materiais/equipamentos de maior tecnologia que ainda não conheciam, 
melhora a assimilação dos conteúdos estudados e do conhecimento adquirido durante as aulas expositivas em sala de aula, correlacionando assim, a teoria com a prática (SCHMITT et al., 2013).

Ao decorrer dos dois períodos em que ocorreu a monitoria 68 alunos foram beneficiados, 30 alunos no período 2013.1 com a aprovação de 27 alunos e 38 alunos no período 2013.2, onde todos foram aprovados. Percebeuse uma procura menor por parte dos alunos para esclarecimento de dúvidas quanto ao conteúdo teórico, e uma maior procura foi evidenciada nos horários destinados a simulação das práticas em laboratório, principalmente nas vésperas dos estágios no hospital.

\section{CONCLUSÃO}

Ao longo deste relato, foram descritos conceitos sobre a experiência da monitoria acadêmica na disciplina de cuidados critícos e sua relevância na formação de futuros enfermeiros. As experiências vivenciadas na monitoria permanecerão impressas naqueles que tiveram a oportunidade de vivenciar essa realidade, os quais experimentaram as primeiras alegrias e decepções do trabalho docente.
Deste modo, revela-se que a monitoria de Cuidados Críticos proporcionou um maior engrandecimento dos graduandos de enfermagem enquanto estudantes e futuros enfermeiros, pois assimilando os conteúdos teóricos com as práticas o processo de ensino-aprendizagem tornase mais dinâmico e de melhor compreensão.

Nós monitoras enfrentamos situações diversas, tanto de alegrias ao colaborar pedagogicamente com 0 aprendizado de alguns alunos e também a decepção com a conduta de outros alunos, as quais se tornaram por vezes desestimuladora, como por exemplo, a procura por parte de alguns alunos apenas para questionar sobre as perguntas que poderiam ser colocadas nas provas. No entato, essas e tantas outras situações foram de fundamental relevância para desabrochar a vocação docente.

A monitoria ao longo dos dois períodos contribuiu para versar a nossa capacidade, em quanto aluno monitor para a concentração, argumentação e domínio sobre o grupo. Ressalta-se também a importância da elaboração dos estudos e pesquisas realizadas, e das trocas de conhecimentos junto ao 
professor orientador e aos alunos monitorados que contribuiu para o nosso engrandecimento intelectual e social, revelando assim novas perspectivas profissionais.

Ressalta-se a necessidade de mais estudo acerca das atividades de monitoria no cenário acadêmico, visto que existem poucos estudos demontrando essa vivência e desses, muitos se encontram ultrapassados devido à data de suas pulblicações. No entanto, os estudos aqui utilizados foram de fundamental relevância para nos dar subsídio para uma melhor visão crítica.

\section{REFERÊNCIAS}

BRASIL. Ministério da Educação. Lei n 9.394 , de 20 de dezembro de 1996. Lei de diretrizes e bases da educação nacional [internet]. Diário Oficial da União, Brasília, 23 de dez, 1996.

Disponível em:

<http://www.planalto.gov.br/ccivil_03/1 eis/19394.htm>. Acesso em 22 ago. 2014.

CARDOSO, Marcelo Viana; ARAÚJO, Rogéria Pimentel. Monitoria acadêmica: relato de experiência em disciplina aplicada a terapia ocupacional.

Cadernos de terapia ocupacional da UFSCar, São Carlos, jan-jun, v.16, n.1, p. 53-57, 2008.
CARVALHO, Isaiane da Silva et al. Monitoria em semiologia e semiotécnica para a enfermagem: um relato de experiência. Ver Enferm UFSM, v. 2, n. 2, 2012. Disponível em:<file:///C:/Documents\%20and\%20S ettings/user/Meus\%20documentos/Dow nloads/3212-27653-1-PB.pdf>. Acesso em 20 ago 2014.

Cervo AL, Berviam PA, Da Silva R. Metodologia Científica. 6 ed. São Paulo: PerasonPretince Hall, 2007.

CONSELHO REGIONAL DE ENFERMAGEM. Código de Ética dos Profissionais de Enfermagem.

Resolução $\mathrm{N}^{\circ} 311$, de 08 de fevereiro de 2007. Rio de Janeiro, jan, 2007.

Disponível em:

$<$ http://se.corens.portalcofen.gov.br/cod igo-de-etica-resolucao-cofen-3112007>. Acesso em 20 ago. 2014.

\section{CONSELHO FEDERAL DE}

ENFERMAGEM. Resolução No 293 de setembro de 2004. Disponível em: <http://www.cofen.gov.br/resoluocofen-2932004_4329.html>. Acesso em 20 ago. 2014.

LINO, Margarete Marques; CALIL, Ana Maria. O ensino de cuidados críticos/intensivos na formação do enfermeiro: momento para reflexão. Rev. esc. enferm. USP, São Paulo, v. 42, n. 4, Dec. 2008.

Disponível em:

$<$ http://www.scielo.br/scielo.php?script =sci_arttext\&pid=S0080-

$62342008000400022 \& \ln \mathrm{g}=\mathrm{en} \& \mathrm{nrm}=\mathrm{iso}$ >. Acesso em 20 Ago. 2014.

NASCIMENTO, Eliane Regina Pereira do; TRENTINI, Mercedes. O cuidado de enfermagem na unidade de terapia intensiva (UTI): teoria humanística de 
Paterson e Zderad. Rev. Latino-Am.

Enfermagem, Ribeirão Preto, v. 12, n. 2, Apr. 2004. Disponível em:

$<$ http://www.scielo.br/scielo.php?script =sci_arttext\&pid=S0104-

$11692004000200015 \& \operatorname{lng}=e n \& n r m=$ iso

>. Acesso em 20 ago. 2014.

SOUZA, Paulo Rogerio Areias de. A importância da monitoria na formação de futuros professores universitários.

In: Âmbito Jurídico, Rio Grande, XII, n. 61, fev, 2009. Disponível

em:<http://www.ambitojuridico.com.br/ site/index.php?n_link=revista_artigos_1 eitura\&artigo_id=5990>. Acesso em 20 ago 2014.

RESOLUÇÃO CSE/UFCG No 09 de 22 de novembro de 2005. Disponível em: < http://www.ufcg.edu.br/ costa/resoluco es/res_12092005.pdf >. Acesso em 15 de jun. 2014.

RESOLUÇÃO CSE/UFCG Nº14 de Junho de 2007. Disponível em:

<http://www.ufcg.edu.br/ costa/resoluc oes/res_16142007.pdf>. Acesso em 15 jun. 2014.

RESOLUÇÃO CSE/UFCG N ${ }^{\circ} 07$ DE ABRIL DE 2009. Disponível em: <http://www.ufcg.edu.br/ costa/resoluc oes/res_16072009.pdf >. Acesso em 15 jun. 2014.

SCHMITT, Márcia Danieli et al. Contribuições da monitoria em semiologia e semiotécnica para a formação do enfermeiro: relato de experiência. Rev UDESC em Ação, v. 7, n. 1, 2013. Disponível em: <http://www.revistas.udesc.br/index. php/udescemacao/article/viewFile/32 64/pdf_37>. Acesso em 20 ago 2014.
UNIVERSIDADE FEDERAL DE CAMPINA GRANDE. Edital PRE $n^{\circ}$ 17/2013. Disponível em: < http://www.ufcg.edu.br:8080/chamadas/ downloads/294605.pdf > . Acesso em 20 ago 2014. 